\title{
Finding Non-Terminating Executions in Distributed Asynchronous Programs
}

\author{
Michael Emmi ${ }^{1 *}$ and Akash $\mathrm{Lal}^{2}$ \\ 1 LIAFA, Université Paris Diderot \\ mje@liafa.univ-paris-diderot.fr \\ 2 Microsoft Research India \\ akashl@microsoft.com
}

\begin{abstract}
Programming distributed and reactive asynchronous systems is complex due to the lack of synchronization between concurrently executing tasks, and arbitrary delay of message-based communication. As even simple programming mistakes have the capability to introduce divergent behavior, a key liveness property is eventual quiescence: for any finite number of external stimuli (e.g., client-generated events), only a finite number of internal messages are ever created.

In this work we propose a practical three-step reduction-based approach for detecting divergent executions in asynchronous programs. As a first step, we give a code-to-code translation reducing divergence of an asynchronous program $P$ to completed state-reachability-i.e., reachability to a given state with no pending asynchronous tasks - of a polynomiallysized asynchronous program $P^{\prime}$. In the second step, we give a code-to-code translation under-approximating completed state-reachability of $P^{\prime}$ by state-reachability of a polynomially-sized recursive sequential program $P^{\prime \prime}(K)$, for the given analysis parameter $K \in \mathbb{N}$. Following Emmi et al. [8]'s delay-bounding approach, $P^{\prime \prime}(K)$ encodes a subset of $P^{\prime}$ 's, and thus of $P$ 's, behaviors by limiting scheduling nondeterminism. As $K$ is increased, more possibly divergent behaviors of $P$ are considered, and in the limit as $K$ approaches infinity, our reduction is complete for programs with finite data domains. As the final step we give the resulting state-reachability query to an off-the-shelf SMT-based sequential program verification tool.

We demonstrate the feasibility of our approach by implementing a prototype analysis tool called ALIVE, which detects divergent executions in several hand-coded variations of textbook distributed algorithms. As far as we are aware, our easy-to-implement prototype is the first tool which automatically detects divergence for distributed and reactive asynchronous programs.
\end{abstract}

\section{Introduction}

The ever-increasing popularity of online commercial and social networks, along with proliferating mobile computing devices, promises to make distributed software an even more pervasive component of technological infrastructure. In a

* Supported by a Fondation Sciences Mathématiques de Paris post-doctoral fellowship. 
distributed program a network of physically separated asynchronous processors coordinate by sending and asynchronously receiving messages. Such systems are challenging to implement because of several uncertainties, including processor timings, message delays, and processor failures. Although simplifying mechanisms such as synchronizers and shared-memory simulation do exist [16], they add significant runtime overhead which can be unacceptable in many situations.

Because of the inherit complexity in distributed asynchronous programming, even subtle design and programming mistakes have the capability to introduce erroneous or divergent behaviors, against which the usual reliability measures are much less effective. The great amount of nondeterminism in processor timings and message delays tends to make errors elusive and hard to reproduce in simulation and testing. The combinatorial explosion incurred by the vast number of processor interleavings and message-buffer contents tends to make formal verification techniques intractable. Though many distributed algorithms are proposed along with manual correctness proofs, key properties such as eventual quiescence - i.e., for any number of external stimuli such as client-generated events, only a finite number of internal network messages are ever createdremain difficult to ensure with automatic techniques. Practically speaking, such properties ensure the eventual construction of network spanning trees [16], the eventual election of network leaders 20, and the eventual acceptance of network peer proposals, e.g., according to the Paxos protocol [15].

In this work we develop an automatic technique to detect violations to eventual quiescence, i.e., executions of distributed systems for which a finite number of external stimuli result in an infinite number of internal messages. Our reduction-based approach works in three steps. First, we reduce the problem of finding nonterminating executions of a given (distributed) asynchronous program $P$ to the problem of computing reachability in a polynomially-sized (distributed) asynchronous program $P^{\prime}$. This reduction is complete for programs with finite data domains, in the sense that an answer to the reachability query on $P^{\prime}$ is a precise answer to the nontermination query on $P$. In the second step, we reduce reachability in $P^{\prime}$ to reachability in a polynomially-sized recursive sequential program $P^{\prime \prime}$-without explicitly encoding the concurrent behavior of $P^{\prime}$ as data in $P^{\prime \prime}$. This step is parameterized by an integer $K \in \mathbb{N}$; for small $K, P^{\prime \prime}$ encodes few concurrent schedules of $P^{\prime}$; as $K$ is increased, $P^{\prime \prime}$ encodes and increasing number of concurrent reorderings, and in the limit as $K$ approaches infinity, $P^{\prime \prime}$ codes all possible behaviors of $P^{\prime}$-and thus $P$. Finally, using existing sequential program verification tools, we check reachability in $P^{\prime \prime}$ : a positive result indicates a nonterminating execution in $P$, though the lack of nonterminating executions in $P$ can only be concluded in the limit as $K$ approaches infinity. Our technique supports fairness, in that we may consider only infinite executions in which no message is ignored forever.

We demonstrate the feasibility of our reduction-based approach by implementing a prototype analysis tool called ALIVE, which detects violations to eventual quiescence in several hand-coded variations to textbook distributed algorithms [16]. Our relatively easy-to-implement prototype leverages existing 
SMT-based program verification tools [14, and as far as we are aware, is the first tool which can automatically detect divergence in distributed asynchronous programs.

To begin in Section 2, we introduce a program model of distributed computation. In Section 3 we describe our reduction to sequential program analysis, and provide code-to-code translations which succinctly encode the reduction. Following in Section 4 we describe our experimental results in analyzing textbook distributed algorithms, and we conclude by discussing related work in Section 5 .

\section{Distributed Asynchronous Programs}

We consider a distributed message-passing program model in which each processor is equipped with a procedure stack and an unordered buffer of pending messages. Initially all processors are idle. When an idle processor's message buffer is nonempty, some message is removed, and a message-dependent task is executed to completion. Each task executes essentially as a recursive sequential program, which besides accessing its own processor's global storage, can post messages to the buffers of any processor, including its own. When a task does complete, its processor again becomes idle, chooses a next pending message to remove, and so on. The distinction between messages and handling tasks is purely aesthetic, and we unify the two by supposing each message is a procedure-and-argument pair. Though in principle many message-passing systems, e.g., in Erlang and Scala, allow reading additional messages at any program point, we have observed that common practice is to read messages only upon completing a prior task 21.

Our choice to model message-passing programs with unordered buffers has two important consequences. First, although some programming models do not ensure messages are received in the order they are sent, others do; our unordered buffer model should be seen as an abstraction of a model with faithful message queues, since ignoring message order allows behaviors infeasible in the queue-ordered model. Second, when message order is ignored, distributed executions are task-serializable-i.e., equivalent to executions where the tasks across all processors execute serially, one after the other. Intuitively this is true because (a) tasks of different processors access disjoint memory, and (b) message posting operations commute with each other. (Message posting operations do not commute when buffers are ordered.) To simulate a distributed system with a single processor we combine each processor's global storage, and ensure each processor's tasks access only their processor-indexed storage. Since serializability implies that single processor systems precisely simulate the behavior of distributed systems, we limit our discussion, without loss of generality, to single-processor asynchronous programs [19]. Appendix A illustrates this mapping to a singleprocessor asynchronous program in more detail.

\subsection{Program Syntax}

Let Procs be a set of procedure names, Vals a set of values, Exprs a set of expressions, Pids a set of processor identifiers, and let $T$ be a type. Figure 1 gives 


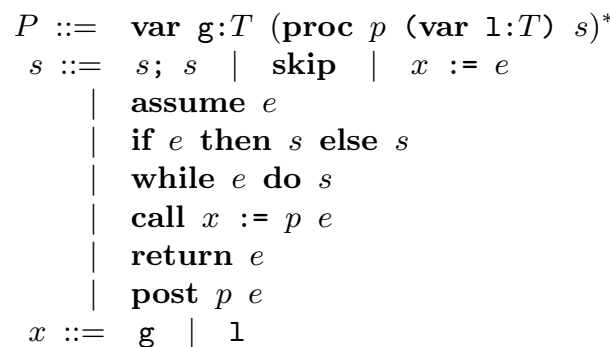

Fig. 1. The grammar of asynchronous message-passing programs $P$. Here $T$ is an unspecified type, and $e$ and $p$ range over expressions and procedure names.

\author{
DISPATCH \\ $\overline{\langle g, \varepsilon, m \cup\{f\}\rangle \rightarrow\langle g, f, m\rangle}$ \\ Complete \\ $\frac{f=\langle\ell, \text { return } e ; s\rangle}{\langle g, f, m\rangle \rightarrow\langle g, \varepsilon, m\rangle}$ \\ Post \\ $\frac{\ell_{2} \in e\left(g, \ell_{1}\right) \quad f=\left\langle\ell_{2}, s_{p}\right\rangle}{\left\langle g,\left\langle\ell_{1}, s_{1}\right\rangle w, m\right\rangle \rightarrow\left\langle g,\left\langle\ell_{1}, s_{2}\right\rangle w, m \cup\{f\}\right\rangle}$
}

Fig. 2. The transition relation $\rightarrow$ of asynchronous message-passing programs.

the grammar of asynchronous message-passing programs. We intentionally leave the syntax of expressions $e$ unspecified, though we do insist Vals contains true and false, and Exprs contains Vals and the (nullary) choice operator $\star$. We say a program is finite-data when Vals is finite.

Each program $P$ declares a single global variable $g$ and a procedure sequence, each $p \in$ Procs having a single parameter 1 and top-level statement denoted $s_{p}$; as statements are built inductively by composition with control-flow statements, $s_{p}$ describes the entire body of $p$. The set of program statements $s$ is denoted Stmts. Intuitively, a post $p$ e statement is an asynchronous call to a procedure $p$ with argument $e$. The assume $e$ statement proceeds only when $e$ evaluates to true, and this statement plays a role in disqualifying executions in our subsequent reductions of Section 3 . The programming language we consider is simple, yet very expressive, since the syntax of types and expressions is left free, and we lose no generality by considering only single global and local variables. Appendix B lists several syntactic extensions which we use in the source-to-source translations of the subsequent sections, and which easily reduce to the syntax of our grammar.

\subsection{Program Semantics}

A (procedure) frame $f=\langle\ell, s\rangle$ is a current valuation $\ell \in$ Vals to the procedurelocal variable 1 , along with a statement $s \in$ Stmts to be executed. (Here $s$ describes the entire body of a procedure $p$ that remains to be executed, and is initially set to $p$ 's top-level statement $s_{p}$; we refer to initial procedure frames $t=\left\langle\ell, s_{p}\right\rangle$ as tasks, to distinguish the frames that populate task buffers.) The set of all frames is denoted Frames. A configuration $c=\langle g, w, m\rangle$ is a current valuation $g \in$ Vals to the processor-global variable $g$, along with a procedure-frame stack $w \in$ Frames* $^{*}$ and a multiset $m \in \mathbb{M}$ [Frames] representing the pending-tasks buffer. The configuration $c$ is called idle when $w=\varepsilon$, and completed when $w=\varepsilon$ and $m=\emptyset$. The set of configurations is denoted Configs. 
Figure 2 defines the transition relation $\rightarrow$ for the asynchronous behavior. (The transitions for the sequential statements are standard, and listed in Appendix C.) The Post rule creates a new frame to execute the given procedure, and places the new frame in the pending-tasks buffer. The COMPLETE rule returns from the final frame of a task, rendering the processor idle, and the Dispatch rule schedules a pending task on the idle processor.

An execution of a program $P$ (from $c_{0}$ ) is a configuration sequence $\xi=c_{0} c_{1} \ldots$ such that $c_{i} \rightarrow c_{i+1}$ for $i \geq 0$; we say each configuration $c_{i}$ is reachable from $c_{0}$. An initial condition $\iota=\left\langle g_{0}, \ell_{0}, p_{0}\right\rangle$ is a global-variable valuation $g_{0} \in$ Vals, along with a local-variable valuation $\ell_{0} \in$ Vals, and a procedure $p_{0} \in$ Procs. A configuration $c=\left\langle g_{0},\left\langle\ell_{0}, s_{p_{0}}\right\rangle, \emptyset\right\rangle$ of a program $P$ is called $\left\langle g_{0}, \ell_{0}, p_{0}\right\rangle$-initial. An execution $\xi=c_{0} c_{1} \ldots$ is called infinitely-often idle when there exists an infinite set $I \subseteq \mathbb{N}$ such that for each $i \in I, c_{i}$ is idle.

Definition 1 (state-reachability). The (completed) state-reachability problem is to determine for an initial condition $\iota$, global valuation $g$, and program $P$, whether there exists a (completed) g-valued configuration reachable in $P$ from $\iota$.

In this work we are interested in detecting non-terminating executions due to asynchrony, rather than the orthogonal problem of detecting whether each individual task may alone terminate. Our notion of non-termination thus considers only executions which return to idle configurations infinitely-often.

Definition 2 (non-termination). The non-termination problem is to determine for an initial condition $\iota$ and a program $P$, whether there exists an infinitelyoften idle execution of $P$ from some $\iota$-initial configuration.

\section{Detecting Non-Termination}

Though precise algorithms for detecting (fair) non-termination in finite-data asynchronous programs are known (see Ganty and Majumdar [10]), the fair nontermination problem is polynomial-time equivalent to reachability in Petri nets, which is an EXPSPACE-hard problem for which only non-primitive recursive algorithms are known. Though worst-case complexity is not necessarily an indication of feasibility on practically-occurring instances, here we are interested in leveraging existing tools designed for more tractable problems whose solutions can be used to incrementally under-approximate non-termination detection; i.e., where for a given analysis parameter $k \in \mathbb{N}$ we can efficiently detect non-termination from an interesting subset $B_{k}$ of program behaviors.

Our strategy is to reduce the problem of detecting non-terminating executions in asynchronous programs to that of completed state-reachability in asynchronous programs. We perform this step using the code-to-code translation of Section 3.1 . and in Section 3.2 we consider extensions to handle fairness. Then, in the second step of Section 3.3 , we apply an incrementally underapproximating reduction from state-reachability in asynchronous programs to state-reachability in sequential program [8, 4, and discharge the resulting program analysis problem using existing sequential analysis tools. 


\subsection{Reduction from Non-Termination to Reachability}

In the first step of our reduction, we use the fact that every infinite execution eventually passes through two configurations $c_{1}$, and then $c_{2}$, such that every possible execution from $c_{1}$ is also possible from $c_{2}$; e.g., when $c_{1}$ and $c_{2}$ are idle configurations with the same global valuation in which all tasks pending at $c_{1}$ are also pending at $c_{2}$. Formally, given two configurations $c_{1}=\left\langle g_{1}, w_{1}, m_{1}\right\rangle$ and $c_{2}=\left\langle g_{2}, w_{2}, m_{2}\right\rangle$ we define the order $c_{1} \preceq c_{2}$ to hold when $g_{1}=g_{2}, w_{1}=w_{2}$, and $m_{1} \subseteq m_{2}{ }^{3}$ An execution $c_{0} c_{1} \ldots$ is called periodic when $c_{i} \preceq c_{j}$ for two idle configurations $c_{i}$ and $c_{j}$ such that $i<\left.j\right|^{4}$ The following lemma essentially exploits the fact that $\preceq$ is a well-quasi-ordering on idle configurations.

Lemma 1. A finite-data program $P$ has an infinitely-often idle execution from $\iota$ if and only if $P$ has a periodic execution from $\iota$.

Proof. Suppose $c_{0} c_{1} \ldots$ is the sequence of idle configurations in an infinitely-often idle execution $\xi$. As the subset order $\subseteq$ on multisets is a well-quasi order, and the domain Vals of global variables is finite, $\preceq$ is a well-quasi order on idle configurations. Thus there exists $i<j$ such that $c_{i} \preceq c_{j}$, so $\xi$ is also periodic.

Supposing $\xi=c_{0} c_{1} \ldots$ is a periodic execution from $\iota$, there exists idle configurations $c_{i}$ and $c_{j}$ of $\xi$ such that $i<j$ and $c_{i} \preceq c_{j}$; let $c_{i}=\left\langle g_{i}, \varepsilon, m_{i}\right\rangle$ and $c_{j}=\left\langle g_{j}, \varepsilon, m_{j}\right\rangle$. Since $g_{i}=g_{j}$ and $m_{i} \subseteq m_{j}$, by definition of $\preceq$, the sequence of execution steps between $c_{i}$ and $c_{j}$ is also enabled from configuration $c_{j}$-we may simply ignore the extra tasks $m_{j} \backslash m_{i}$ pending in $c_{j}$. For any $k, l \in \mathbb{N}$ and task buffer $m \in \mathbb{M}\left[\right.$ Frames] such that $k<l<|\xi|$, let $\xi_{k, l}^{m}$ be the sequence of configurations $c_{k} c_{k+1} \ldots c_{l-1}$ of $\xi$, each with additional pending tasks $m$. Furthermore, let $k \cdot m$ be the multiset union of $k$ copies of $m$. Letting $m=m_{j} \backslash m_{i}$, then $\xi_{0, i} \xi_{i, j} \xi_{i, j}^{m} \xi_{i, j}^{2 m} \xi_{i, j}^{3 m} \ldots$ is an infinitely-often idle execution from $\iota$ which periodically repeats the same transitions used to construct $\xi$ between $c_{i}$ and $c_{j}$.

We reduce the detection of periodic executions to completed state reachability in asynchronous programs. Essentially, such a reduction must determine multiset inclusion between the unbounded task buffers at two idle configurations; i.e., for some idle configuration $c_{i}=\left\langle g_{i}, \varepsilon, m_{i}\right\rangle$ reachable in an execution $c_{0} c_{1} \ldots$, there exists $j>i$ such that $c_{j}=\left\langle g_{j}, \varepsilon, m_{j}\right\rangle$ with $g_{i}=g_{j}$ and $m_{i} \subseteq m_{j}$. As the set $m_{i}$ of pending tasks at $c_{i}$ is unbounded, any reduction cannot hope to store arbitrary $m_{i}$ for later comparison with $m_{j}$ using finite-domain program variables.

Our reduction determines the correspondence between unbounded task buffers in the source program using only finite-domain program variables by leveraging the task buffers of the target program. For each instance of a task $t$ which is pending in $c_{i}$, we post an additional task pro $(t)$ when $t$ is posted; for each task $t$ pending in $c_{j}$, we either post an additional task anti $(t)$ instead of $t$, or we post nothing, to handle the case where $t$ is never dispatched. We then check that for each executed pro $(t)$ a matching anti $(t)$ is also executed, and that at

\footnotetext{
${ }^{3}$ Here $\subseteq$ is the multiset subset relation.

${ }^{4}$ As our definition of $\preceq$ only relates configurations with equal global valuations, our notion of periodic is only complete for finite-data programs.
} 


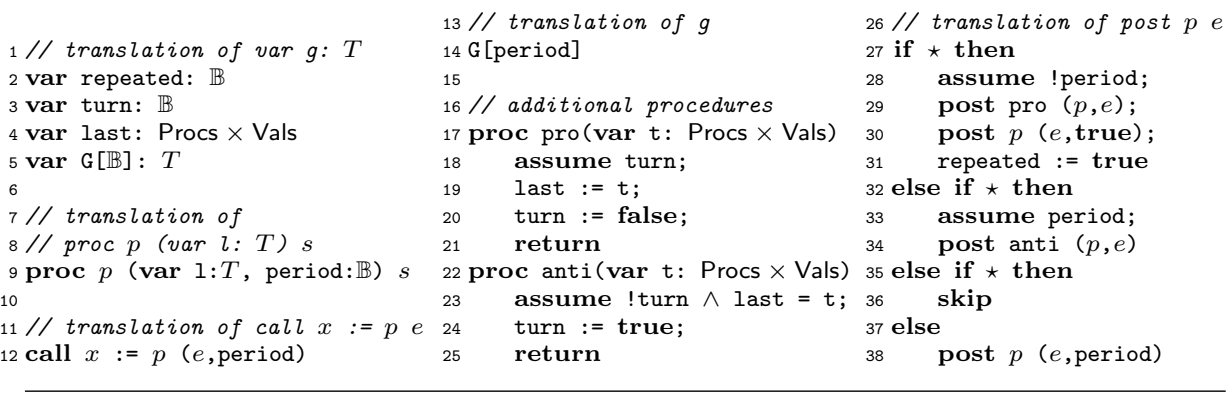

Fig. 3. The translation $((P))_{\mathrm{nt}}$ of an asynchronous program $P$.

some point no pro $(t)$ nor anti $(t)$ tasks are pending. By considering executions which alternate between tasks of $\left\{\operatorname{pro}(t): t \in m_{i}\right\}$ and $\left\{\operatorname{anti}(t): t \in m_{j}^{\prime}\right\}$-where $m_{j}^{\prime} \subseteq m_{j}$ such that $m_{j} \backslash m_{j}^{\prime}$ correspond to the dropped tasks - we can ensure each instance of an $m_{i}$ task has a corresponding instance in $m_{j}$, storing only the last encountered pro $(t)$ task, for $t \in m_{i}$.

Figure 3 lists our code-to-code translation $((P))_{n t}$ reducing non-termination in an asynchronous program $P$ to completed state reachability in the asynchronous program $((P))_{n t}$. Besides the auxiliary variable last used to store the last encountered $\operatorname{pro}(t)$ task, for $t \in m_{i}$, we introduce Boolean variables repeated, to signal whether $m_{i}$ is non-empty, and turn, to signal whether an anti $(t)$ task has been executed since the last executed pro $(t)$ task. We also divide the execution of tasks into two phases by introducing a task-local Boolean variable period. The first phase (!period) corresponds to the execution $c_{0} c_{1} \ldots c_{i}$, while the second phase (period) corresponds to $c_{i+1} c_{i+2} \ldots c_{j}$. Initially pending tasks occur in the first non-period phase. Then each time a new task $t$ is posted, a non-deterministic choice is made for whether $t$ will execute in the non-period phase, in the period phase, or never.

Finally, to determine which finite asynchronous executions prove the existence of infinite asynchronous executions, we define the predicate $\varphi_{\text {nt }}$ over initial conditions $\iota$ and configuration $c$ as

$$
\varphi_{\mathrm{nt}}(\iota, c) \stackrel{\text { def }}{=}\left\{\begin{aligned}
\text { true } & \text { when } \neg \text { repeated }(\iota) \text { and } \operatorname{turn}(\iota) \\
& \text { and repeated }(c) \text { and } \operatorname{turn}(c) \\
& \text { and } \mathrm{G}[0](c)=\mathrm{G}[1](\iota)=\mathrm{G}[1](c) \\
\text { false } & \text { otherwise, }
\end{aligned}\right.
$$

along with the mapping $\vartheta_{\text {nt }}$ which projects the initial conditions of $((P))_{\mathrm{nt}}$ to those of $P$, as $\vartheta_{\text {nt }}(\langle g, \ell, p\rangle) \stackrel{\text { def }}{=}\left\langle g^{\prime}, \ell^{\prime}, p^{\prime}\right\rangle$ when $\mathrm{g}\left(g^{\prime}\right)=\mathrm{G}[0](g), I\left(\ell^{\prime}\right)=1(\ell)$, and $p^{\prime}=p$. Essentially, in any completed configuration $c$ reachable from $\iota$ satisfying $\varphi_{\text {nt }}(\iota, c)$, we know that some task has executed during the period (since repeated evaluates to true), and that for each task pending at the beginning of the period, an identical task is pending at the end of the period (since turn evaluates to true, and there are no pending tasks in $c$ ). Finally, the conditions on the global 
variable $\mathrm{G}$ ensure that the beginning and end of each period reach the same global valuation.

Lemma 2. A finite-data program $P$ has an infinitely-often idle execution from $\iota_{0}$ if and only if a completed configuration $c$ is reachable in $((P))_{n t}$ from some $\iota$ such that $\varphi_{\mathrm{nt}}(\iota, c)=$ true and $\vartheta_{\mathrm{nt}}(\iota)=\iota_{0}$.

Proof. For the forward direction, by Lemma 1, $P$ also has a periodic execution $\xi=\xi_{0, i} \xi_{i, j} \xi_{j, \omega}$ from $\iota_{0}$-where $\xi_{k, l} \stackrel{\text { def }}{=} c_{k} c_{k+1} \ldots c_{l-1}$ for $k<l<|\xi|$-and $c_{i} \preceq c_{j}$ for idle configurations $c_{i}=\left\langle g, \varepsilon, m_{1}\right\rangle$ and $c_{j}=\left\langle g, \varepsilon, m_{2}\right\rangle$. We build an execution $\xi^{\prime}=\xi_{0, i}^{\prime} \xi_{i, j}^{\prime} \xi_{\text {match }}$ of $((P))_{\mathrm{nt}}$ such that

- the configurations $c_{k}^{\prime}$ of $\xi_{0, i}^{\prime}$ correspond to configurations $c_{k}$ of $\xi_{0, i}$, with $\mathrm{g}\left(c_{k}\right)=\mathrm{G}[0]\left(c_{k}^{\prime}\right), \mathrm{G}[1]\left(c_{k}^{\prime}\right)=g$,

- the configurations $c_{k}^{\prime}$ of $\xi_{i, j}^{\prime}$ correspond to configurations $c_{k}$ of $\xi_{i, j}$, with $\mathrm{g}\left(c_{k}\right)=\mathrm{G}[1]\left(c_{k}^{\prime}\right)$ and $\mathrm{G}[0]\left(c_{k}^{\prime}\right)=g$,

- the pending tasks of each configuration $c_{k}^{\prime}$ of $\xi_{0, j}^{\prime}$, excluding pro and anti tasks, are contained within those of $c_{k}$,

- the local valuations of each configuration $c_{k}^{\prime}$ of $\xi_{0, i}^{\prime}$ (resp., of $\xi_{i, j}^{\prime}$ ) match those of $c_{k}$, except period evaluates to 0 (resp., to 1 ) in every frame of $c_{k}^{\prime}$, and

- the sequence $\xi_{\text {match }}$ alternately executes pro and anti tasks such that each pro $(t)$ task is followed by a matching anti $(t)$ task.

It follows that we can construct such a $\xi^{\prime}$ which reaches a completed configuration $c$ from some $\iota$ such that $\varphi_{\mathrm{nt}}(\iota, c), \vartheta_{\mathrm{nt}}(\iota)=\iota_{0}$, and $\mathrm{G}[0](c)=\mathrm{G}[1](c)=g$.

For the backward direction, the reachability of a completed configuration $c$ of $((P))_{\mathrm{nt}}$ from $\iota$ such that $\varphi_{\mathrm{nt}}(\iota, c)$ implies that there exists a periodic execution $\xi=c_{0} c_{1} \ldots$ of $P$; in particular, there exist configurations $c_{i} \preceq c_{j}$ of $\xi$ with $i<j$, and which have the global valuations $\mathrm{g}\left(c_{i}\right)=\mathrm{g}\left(c_{j}\right)=\mathrm{G}[0](c)=\mathrm{G}[1](c)$ reached at the end of each period of $((P))_{\mathrm{nt}}$ 's execution, and the set of pending tasks $m$ in $c_{i}$ are those second-period tasks posted by $((P))_{\mathrm{nt}}$ from first-period tasks. Since the set of tasks posted and pending by the end of the second period must contain $m$ - otherwise unexecutable pro tasks would remain pending - we can construct a run where the pending tasks of $c_{j}$ contain the pending tasks of $c_{i}$, and so $P$ has a periodic execution. By Lemma 1 we conclude that $P$ also has an infinitely-often idle execution.

\subsection{Ensuring Scheduling Fairness}

In many classes of asynchronous systems there are (at least) two sensible notions of scheduling fairness against which to determine liveness properties: an infinite execution is called strongly-fair if every infinitely-often enabled transition is fired infinitely often, and weakly-fair if every infinitely-often continuously enabled transition is fired infinitely often. In our setting where asynchronous tasks execute serially from a task buffer, weak fairness becomes irrelevant; while one task executes no other transitions are enabled, and when idle (i.e., while no tasks are executing), all pending tasks become enabled. Furthermore once a task is posted, 
it becomes pending, and it is thus enabled in all subsequent idle configurations until dispatched. We thus define fairness according to what is normally referred to as strong fairness: an execution is fair when each infinitely-often posted task is infinitely-often dispatched.

To extend our reduction so that only fair infinite executions are considered we make two alterations to the translation of Figure 3. First, on Line 36 we replace skip with assume period; this ensures participation of all tasks pending at the beginning of each period. Second, we add auxiliary state to ensure at least one instance of each task posted during the period is dispatched. This can be encoded in various ways; for instance, we can add two arrays dropped and dispatched of index type Procs $\times$ Vals and element type $\mathbb{B}$ that indicate whether each task has been dropped/dispatched during the period phase (i.e., where the local variable period evaluates to true). Initially dropped $[t]=\operatorname{dispatched}[t]=$ false for all $t \in$ Procs $\times$ Vals. Each time a post to task $t$ is dropped during the period phase (i.e., Line 36) we set dropped [t] to true, and each time task $t$ is executed during the period phase (i.e., Line 38 when period is true) we set dispatched $[t]$ to true. (Note that we need not consider the non-post of $t$ on Line 34 as dropped, since $t$ is necessarily dispatched during the period phase; otherwise there would remain a pending anti $(t)$ task.) Finally, we add to our reachability query the predicate $\forall t$.dropped $[t] \Rightarrow$ dispatched $[t]$, thus ensuring that when all asynchronous tasks have completed the only dropped tasks have been dispatched during the period.

Alternatively, we may also encode this fairness check by posting auxiliary dropped and dispatched tasks to the task buffer, in place of using the dropped and dispatched arrays. Essentially for each task $t$ dropped during the period phase on Line 36 we add post dropped $(t)$, and for each task $t$ posted into the period phase we add post dispatched $(t)$. Then, using a single additional variable of type Procs $\times$ Vals we ensure that for every executed dropped $(t)$ task some dispatched $(t)$ task also executes; a single variable suffices for this check because we may consider only schedules where all dropped $(t)$ and dispatch(t) tasks execute contiguously for each $t$.

\subsection{Delay-Bounded Reachability}

Following the reduction from (fair) nontermination, we are faced with a highlycomplex problem: determining completed state-reachability in finite-data programs is polynomial-time equivalent to computing exact reachability in Petri nets (i.e., such that all places representing pending tasks are empty), or alternatively in vector addition systems (i.e., such that all vector components counting pending tasks are zero). Though these problems are known to be decidable, there is no known primitive-recursive upper complexity bound.

Rather than dealing with such difficult problems, our strategy is to consider only a restricted yet interesting set of actual program behaviors. Following Emmi et al. [8]'s delay-bounding scheme, we equip some deterministic task scheduler with the ability to deviate from its deterministic schedule only a bounded number of times (per task). As this development is very similar to Emmi et al. [8]'s, we 


\begin{tabular}{l|cccr} 
& \multicolumn{4}{c}{} \\
Example & bug? & $K$ & $N$ & time $(\mathrm{s})$ \\
\hline PingPong & $\sqrt{ }$ & 1 & 5 & 5.32 \\
PingPong-mod2 & $\sqrt{ }$ & 2 & 5 & 19.01 \\
PingPong-mod2-1md & $\times$ & 1 & 5 & 4.94 \\
PingPong-mod3 & $\sqrt{ }$ & 3 & 5 & 86.61 \\
PingPong-mod3-1md & $\times$ & 2 & 5 & 23.53 \\
PingPong-mod3-2md & $\times$ & 1 & 5 & 4.66 \\
PingPongPung & $\sqrt{ }$ & 2 & 5 & 111.92 \\
PingPongPung-1md & $\times$ & 1 & 5 & 19.87 \\
\hline SpanningTree-bug & $\sqrt{ }$ & 1 & 5 & 165.19 \\
SpanningTree-correct & $\times$ & 2 & 3 & 28.80 \\
Bfs-bug & $\sqrt{ }$ & 1 & 5 & 286.95 \\
Bfs-correct & $\times$ & 2 & 3 & 32.15 \\
BellmanFord-bug & $\sqrt{ }$ & 1 & 5 & 303.98 \\
BellmanFord-correct & $\times$ & 2 & 3 & 33.74 \\
\hline Paxos-bug-individual & $\sqrt{ }$ & 2 & 2 & 67.72 \\
Paxos-bug-competition & $\sqrt{ }$ & 2 & 2 & $\mathrm{~T} / \mathrm{O}$ \\
\hline
\end{tabular}

Fig. 5. Experimental results with Alive. Here $K$ indicates the delay-bound, and $N$ the recursion-depth bound.
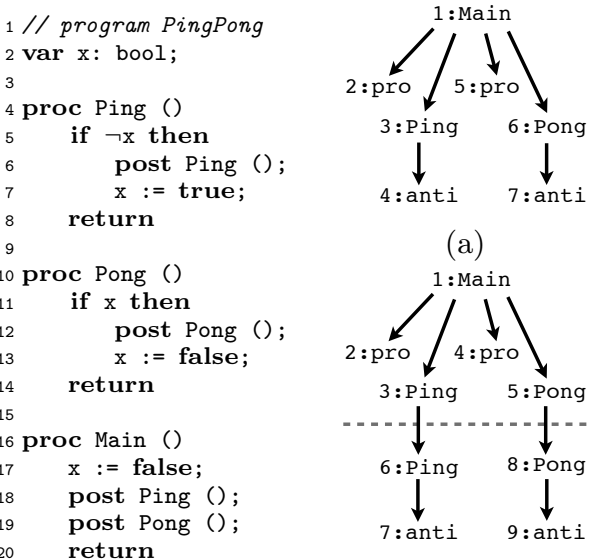

(a)

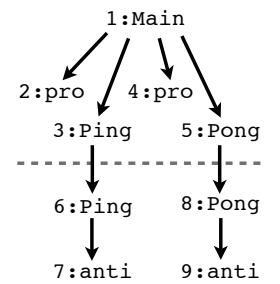

(b)

Fig. 6. The PingPong program, along with asynchronous executions of the translations ((PingPong) $)_{n t}$ (a) and $((\text { PingPong-mod2 }))_{n t}$ (b). Task order is indicated by numeric prefixes; the dotted line indicates delaying.

an execution using at most $K$ delay operations, per task). AsYNCCHECKER essentially performs a variation of our delay-bounded translation of Section 3.3 which results in a sequential Boogie program - and hands the resulting program $P^{\prime \prime}$ to the CorRal SMT-based bounded model checker 14 to detect assertion violations.

Our implementation is able to find (fair) non-terminating executions in several toy examples, and handed-coded implementations of textbook distributed algorithms [16]; the source code of our examples can be found online [7. Figure 5 summarizes our experiments on three families of examples which we discuss below: the PingPong family of toy examples, the SpanningTree family of textbook examples, and variations on Lamport's Paxos algorithm [15. For each family, Figure 5 lists both "buggy" variations (i.e., those with infinite executions) and "correct" variations (those without infinite executions - at least up to the given delay bound). In each case the delay bound is given by $K$, and a recursion bound is given by $N$; our back-end bounded model checker CORRAL only explores executions in which the procedure stack never contains more than $N$ frames of any procedure, for a given recursion bound $N \in \mathbb{N}$. Note that our implementation is a simple unoptimized prototype; the running times are simply listed as a validation that our reduction is feasible. 


\subsection{PingPong}

As a simple example of a non-terminating asynchronous program, consider the PingPong program of Figure 6. Initially the Main procedure initializes the Boolean variable $\mathrm{x}$ to false and posts asynchronous calls to Ping and Pong. When Ping executes and $\mathrm{x}$ is false, then Ping posts a subsequent call to Ping, and sets $\mathrm{x}$ to true; otherwise Ping simply returns. Similarly, when Pong executes and $\mathrm{x}$ is true, then Pong posts a subsequent call to Pong, and sets $\mathrm{x}$ to false; otherwise Pong simply returns. This program has exactly one non-terminating execution: that where the pending instances to Ping and Pong execute in alternation. This execution is periodic, as the configuration where $\mathrm{x}=$ false, and both Ping and Pong have a single pending instance, is encountered infinitely often.

Figure 6a depicts an execution of the program resulting from our translation (Section 3.1) of the PingPong program. Following our translation, the Main procedure takes the branch of Line 28 in Figure 3, posting both pro(Ping) and Ping, then both pro(Pong) and Pong. Without using any delay operations, the scheduler encoded by ASYNCCHECKER executes the posted tasks in depthfirst order over the task-creation tree [8, 9]. Thus following Main, pro(Ping) executes, then Ping, followed by anti(Ping). Subsequently, pro(Pong), Pong, and anti (Pong) execute, in that order. Luckily this execution provides a witness to nontermination without spending a single delay.

Our experiments include several variations of this example. The -mod2 and -mod3 variations add an integer variable $i$ which is incremented (modulo 2, resp., 3) by each call of Ping. The addition of this counter complicates the search for a repeated configuration, since besides the global variable $\mathrm{x}$ and pending tasks Ping and Pong, the value of $i$ must also match in the repeating configuration. This addition also increases the number of delay operations required to discover an infinite execution, as the depth-first task scheduler without delaying considers only executions where all Ping tasks execute before all Pong tasks - see Figure 6b; since, for instance, modulo 2 incrementation requires two of each Ping and Pong tasks to return to a repeating configuration (i.e., with $i=0$ ), the second Ping task must delay in order to occur after the first Pong task. In the $-1 \mathrm{md}$ and $-2 \mathrm{md}$ variations, we reduce the budget of task delaying, and observe that indeed the additional delay budgets are required to witness nonterminating executions. The PingPongPung variation is an even more intricate variation in which each task (i.e., Ping, Pong, or Pung) posts a different task.

\subsection{SpanningTree}

In Figure 7 we consider two examples of distributed algorithms taken from the textbook of Lynch [16], and modified to introduce nonterminating executions. Essentially, SpanningTree attempts to compute a spanning tree for an arbitrary network by building a parent relation from message broadcasts. When the parent link is established asynchronously there exist (unfair) executions in which nodes cyclically propagate their search messages without ever establishing the parent relation. The BellmanFord algorithm is a generalization of SpanningTree 
in case their initial proposal is not answered within a timeout (Line 12), when NOTIFY_DECLINED is false. Then even an individual proposer may repeatedly propose new values just before receiving the acceptors' proposal_OK messages. The acceptors, in turn, may continue to increment their prepared values, such that previously agreed proposals will no longer be accepted (see the condition on Line 40). Even preventing such behavior by assuming the proposers only submit new proposals upon the reception of declined messages (i.e., suppose NOTIFY_DECLINED is true), fair nonterminating executions may still arise by competition between two or more proposers; for instance where two proposers continuously outbid the other before either's proposal has been accepted.

Since each subsequent proposal in the Paxos algorithm proposed an increasingly large number, strictly speaking our detection algorithm will not discover such nonterminating executions, since the same values of proposal and prepared will not be encountered twice. Essentially we must extend our well-quasi-ordering of Section 3.1 by relaxing the equality on global state valuations to a well-quasiordering which is compatible with the program's transition relation. For the purpose of our experiments, we have encoded manually such an order $\preceq^{\prime}$ for our variations on the Paxos algorithm; the order relates global valuations $g_{1} \preceq^{\prime} g_{2}$ when there exists some $\delta \in \mathbb{N}$ such that the values of proposal for proposing processes, and prepared for accepting processes, in $g_{1}$ and $g_{2}$ uniformly increase by $\delta$, and all other variables in $g_{1}$ and $g_{2}$ are equal. With this small manual effort, Alive is able to discover the "individual" nonterminating execution described above, and while ALIVE can also detect the "competing" nonterminating execution in theory, AsYNCCHECKER times out on the reachability check after 30 minutes.

\section{Related Work}

Contrary to much work on sequential program (non)termination detection [5, 11, less attention has been paid to concurrent programs, where nontermination can arise from asynchronous interaction rather than diverging data values. Though both Cook et al. [6] and Popeea and Rybalchenko [17] have proposed techniques to prove termination in multithreaded programs, failure to prove termination does not generally indicate the existence of nonterminating executions. In very recent work, Atig et al. [1] suggest compositional nontermination detection for multithreaded programs based on bounded context-switch; their technique detects infinite executions between a group of interfering, and each non-terminating, threads. Our approach is orthogonal, as we detect infinite executions in which every task terminates; nontermination arises from the never-ending creation of new tasks. Technically, while Atig et al. 11 explore the behaviors between statically-known threads, our problem is to detect the repetition of an unbounded set of dynamically-created tasks.

Our reduction-based technique follows a recent trend of compositional translations to sequential program analysis by considering bounded program behaviors. Based on the notion of bounded context-switch [18, Lal and Reps [13] proposed 


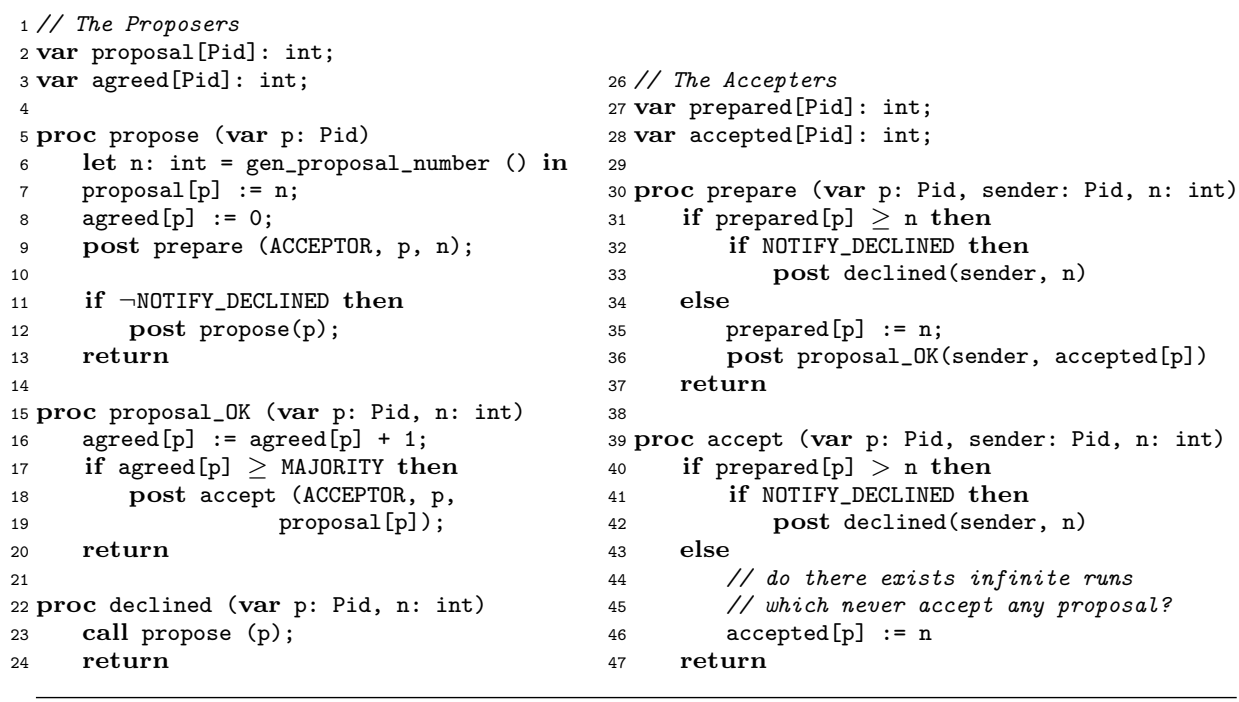

Fig. 8. A basic variation of the Paxos distributed algorithm; for simplicity we suppose there is only a single accepting process named ACCEPTOR.

a reduction from detecting safety violations in multithreaded programs (with a finite number of statically-known threads) to detecting safety violations in sequential programs; shortly after La Torre et al. [12] extended this result to handle an arbitrary number of parametric threads, which was further extended by Emmi et al. 8 to handle dynamic thread creation-including the case of task-buffer based "asynchronous programs" [19]. More recently Bouajjani and Emmi [3] proposed a reduction from safety violations in distributed asynchronous programs with ordered message queues. Thus far, only the recent (yet orthogonal-see above) work of Atig et al. 1] considers liveness properties such as nontermination.

Finally, although reductions from fair nontermination of task-buffer based finite-data asynchronous programs (alternatively, Petri nets) are known- e.g., by encoding into Petri net path logic formalæ [10] our encoding into asynchronous programs is original, and takes advantage of existing program analysis tools with efficient under-approximating exploration strategies. Technically, Ganty and Majumdar [10]'s encoding uses constraints on marking-valued variables to ensure that each task pending at the beginning of a repeating period is re-posted and pending at the period's end; a path-logic solver must then determine satisfiability under those constraints. Our encoding handles the matching of pre- and postperiod pending tasks directly; we pose an asynchronous program reachability query on a program whose additional tasks block executions in which pre- and post-period tasks cannot be matched. 


\section{Conclusion}

We have proposed a practical reduction-based algorithm for detecting divergent executions in distributed asynchronous programs. By incrementally increasing possible task reordering, our approach explores an increasing number of possiblydivergent behaviors with increasing analysis cost, and any possibly-divergent behavior is considered at some cost. By reducing divergence of distributed asynchronous programs to assertion violation in sequential programs, our approach leverages efficient off-the-shelf sequential program analysis tools. Using our prototype tool, Alive, we demonstrate that the approach is able to find divergent executions in modified versions of typical textbook distributed algorithms.

\section{References}

[1] M. F. Atig, A. Bouajjani, M. Emmi, and A. Lal. Detecting fair nontermination in multithreaded programs. In $C A V$ '12: Proc. 24th International Conference on Computer Aided Verification, LNCS. Springer, 2012.

[2] M. Barnett, K. R. M. Leino, M. Moskal, and W. Schulte. Boogie: An intermediate verification language. http://research.microsoft.com/en-us/ projects/boogie/.

[3] A. Bouajjani and M. Emmi. Bounded phase analysis of message-passing programs. In TACAS '12: Proc. 18th International Conference on Tools and Algorithms for the Construction and Analysis of Systems, LNCS. Springer, 2012.

[4] A. Bouajjani, M. Emmi, and G. Parlato. On sequentializing concurrent programs. In $S A S$ '11: Proc. 18th International Symposium on Static Analysis, volume 6887 of LNCS, pages 129-145. Springer, 2011.

[5] B. Cook, A. Podelski, and A. Rybalchenko. Termination proofs for systems code. In PLDI '06: Proc. ACM SIGPLAN 2006 Conference on Programming Language Design and Implementation, pages 415-426. ACM, 2006.

[6] B. Cook, A. Podelski, and A. Rybalchenko. Proving thread termination. In PLDI '07: Proc. ACM SIGPLAN 2007 Conference on Programming Language Design and Implementation, pages 320-330. ACM, 2007.

[7] M. Emmi and A. Lal. Finding non-terminating executions in distributed asynchronous programs. May 2012. http://hal.archives-ouvertes.fr/ hal-00702306/.

[8] M. Emmi, S. Qadeer, and Z. Rakamarić. Delay-bounded scheduling. In POPL '11: Proc. 38th ACM SIGPLAN-SIGACT Symposium on Principles of Programming Languages, pages 411-422. ACM, 2011.

[9] M. Emmi, A. Lal, and S. Qadeer. Asynchronous programs with prioritized task-buffers. Technical Report MSR-TR-2012-1, Microsoft Research, 2012.

[10] P. Ganty and R. Majumdar. Algorithmic verification of asynchronous programs. CoRR, abs/1011.0551, 2010. http://arxiv.org/abs/1011.0551.

[11] A. Gupta, T. A. Henzinger, R. Majumdar, A. Rybalchenko, and R.-G. Xu. Proving non-termination. In POPL '08: Proc. 35th ACM SIGPLAN-SIGACT 
Symposium on Principles of Programming Languages, pages 147-158. ACM, 2008.

[12] S. La Torre, P. Madhusudan, and G. Parlato. Model-checking parameterized concurrent programs using linear interfaces. In $C A V$ '10: Proc. 22nd International Conference on Computer Aided Verification, volume 6174 of LNCS, pages 629-644. Springer, 2010.

[13] A. Lal and T. W. Reps. Reducing concurrent analysis under a context bound to sequential analysis. Formal Methods in System Design, 35(1):73-97, 2009.

[14] A. Lal, S. Qadeer, and S. Lahiri. Corral: A solver for reachability modulo theories. In $C A V$ '12: Proc. 24th International Conference on Computer Aided Verification, LNCS. Springer, 2012.

[15] L. Lamport. The part-time parliament. ACM Trans. Comput. Syst., 16(2): 133-169, 1998.

[16] N. A. Lynch. Distributed Algorithms. Morgan Kaufmann, 1996. ISBN 1-55860-348-4.

[17] C. Popeea and A. Rybalchenko. Compositional termination proofs for multithreaded programs. In TACAS '12: Proc. 18th International Conference on Tools and Algorithms for the Construction and Analysis of Systems, LNCS. Springer, 2012.

[18] S. Qadeer and J. Rehof. Context-bounded model checking of concurrent software. In TACAS '05: Proc. 11th International Conference on Tools and Algorithms for the Construction and Analysis of Systems, volume 3440 of $L N C S$, pages 93-107. Springer, 2005.

[19] K. Sen and M. Viswanathan. Model checking multithreaded programs with asynchronous atomic methods. In $C A V^{\prime} 06$ : Proc. 18th International Conference on Computer Aided Verification, volume 4144 of LNCS, pages 300-314. Springer, 2006.

[20] H. Svensson and T. Arts. A new leader election implementation. In Erlang '05: Proc. 2005 ACM SIGPLAN Workshop on Erlang, pages 35-39. ACM, 2005.

[21] F. Trottier-Hebert. Learn you some Erlang for great good! http: //learnyousomeerlang.com/.

\section{A Distributed to Shared-Memory}

We illustrate the process of going from a distributed program to a single-processor asynchronous program in Figure 9. The figure shows the generic structure of a distributed program with $N$ machines. Each machine has an unordered buffer of pending messages and memory that is not shared with any other machine. Here, we capture the memory of a machine using a single global variable $\mathrm{g}_{i}$. The computation on a machine is an event-loop that picks any message from its buffer (if any) and processes it. The processing of a message can send other messages, but is not allowed to receive any message. The figure also shows a client that can send messages to one of these machines to initiate computation in the distributed system. 


\begin{tabular}{|c|c|c|c|}
\hline \multirow{3}{*}{$\begin{array}{l}\text { // Machine } i \\
\text { var } \mathrm{g}_{i}: T \\
{\text { proc } \operatorname{main}_{i}()} \\
\text { init }_{i}() ; \\
\text { while }(\mathrm{m}=\operatorname{receive}()) \operatorname{process}_{i}(\mathrm{~m}) ; \\
\text { return }\end{array}$} & $\begin{array}{l}\operatorname{proc}_{\operatorname{process}_{i}(m)} \\
\ldots \ldots \\
\operatorname{send}\left(j, m^{\prime}\right)\end{array}$ & $\begin{array}{l}\text { // Shared memory } \\
\operatorname{var} \mathrm{g}_{1}, \mathrm{~g}_{2}, \cdots, \mathrm{g}_{N}: T\end{array}$ & $\begin{array}{l}\operatorname{proc}_{\operatorname{process}_{i}(\mathrm{~m})} \\
\ldots \text { post process }\left(\mathrm{m}^{\prime}\right)\end{array}$ \\
\hline & return & $\begin{array}{l}\operatorname{proc} \operatorname{main}() \\
\quad \operatorname{init}_{1}()\end{array}$ & return \\
\hline & $\begin{array}{l}\text { // External client } \\
\text { proc client () } \\
\text { send }(1, \mathrm{~m}) \\
\text { return }\end{array}$ & $\begin{array}{l}\operatorname{init}_{N}() ; \\
\text { // start client } \\
\text { post client (); } \\
\text { return }\end{array}$ & $\begin{array}{l}\text { proc client() } \\
\text { post process }{ }_{1}(\mathrm{~m}) \\
\text { return }\end{array}$ \\
\hline
\end{tabular}

Fig. 9. (a) A generic distributed program with $N$ machines. (b) A semantically equivalent shared-memory asynchronous program with a single task buffer.

The translated asynchronous program executes on a single processor and has a single unordered buffer of pending tasks. Its global memory is a union of the memory of each machine. The main procedure initializes all machines and starts the client. The sending of a message is simply a post to the task buffer of the asynchronous program. It is easy to see that the two programs have the same semantics.

\section{B Syntactic Extensions Used in Our Code Translations}

The following syntactic extensions are reducible to the original program syntax of Section 2.1. Here we freely assume the existence of various type- and expressionconstructors. This does not present a problem since our program semantics does not restrict the language of types nor expressions.

Multiple types. Multiple type labels $T_{1}, \ldots, T_{j}$ can be encoded by systematically replacing each $T_{i}$ with the sum-type $T=\sum_{i=1}^{j} T_{i}$. This allows local and global variables with distinct types.

Multiple variables. Additional variables $\mathrm{x}_{1}: T_{1}, \ldots, \mathrm{x}_{j}: T_{j}$ can be encoded with a single record-typed variable $\mathrm{x}: T$, where $T$ is the record type

$$
\left\{\mathrm{f}_{1}: T_{1}, \ldots, \mathrm{f}_{j}: T_{j}\right\}
$$

and all occurrences of $\mathrm{x}_{i}$ are replaced by $\mathrm{x} . \mathrm{f}_{i}$. When combined with the extension allowing multiple types, this allows each procedure to declare any number and type of local variable parameters, distinct from the number and type of global variables.

Local variable declarations. Additional (non-parameter) local variable declarations var I': $T$ to a procedure $p$ can be encoded by adding $I^{\prime}$ to the list of parameters, and systematically adding an initialization expression (e.g., the choice expression

$\star$, or false) to the corresponding position in the list of arguments at each call site of $p$ to ensure that l' begins correctly (un)initialized. 
Unused values. Call assignments call $\mathrm{x}:=p e$, where $\mathrm{x}$ is not subsequently used, can be written as call _ $:=p e$, where _ $: T$ is an additional unread local variable, or simpler yet as call $p e$.

Unused branches. if $e$ then $s$ else skip is abbreviated by if $e$ then $s$.

Increment. Increment operations $x++$ are encoded as $x:=x+1$.

Let bindings. Let bindings of the form let $\mathrm{x}: T=e$ in can be encoded by declaring $\mathrm{x}$ as a local variable var $\mathrm{x}$ : $T$ immediately followed by an assignment $\mathrm{x}:=e$. This construct is used to explicate that the value of $\mathrm{x}$ remains constant once initialized. The binding let $\mathrm{x}: T$ in is encoded by the binding let $\mathrm{x}: T=\star$ in where $\star$ is the choice expression.

Arrays. Finite arrays with $j$ elements of type $T$ can be encoded as records of type $\left\{\mathrm{f}_{1}: T, \ldots, \mathrm{f}_{j}: \mathrm{T}\right\}$, where $\mathrm{f}_{1} \ldots \mathrm{f}_{j}$ are fresh names. Occurrences of terms a [i] are replaced by a. $f_{i}$, and array-expressions $\left[e_{1}, \ldots, e_{j}\right]$ are replaced by record-expressions $\left\{f_{1}=e_{1}, \ldots, f_{j}=e_{j}\right\}$.

\section{Sequential Program Semantics}

For expressions without program variables, we assume the existence of an evaluation function $\llbracket \cdot \rrbracket_{\mathrm{e}}$ : Exprs $\rightarrow \wp($ Vals $)$ such that $\llbracket \star \rrbracket_{\mathrm{e}}=$ Vals. For convenience we define $e(g, \ell) \stackrel{\text { def }}{=} \llbracket e[g / g, \ell / 1] \rrbracket_{\mathrm{e}}$ to evaluate the expression $e$ in a global valuation $g$ by substituting the current values for variables $g$ and 1 . As these are the only program variables, the substituted expression $e[g / g, \ldots]$ has no free variables.

To further reduce clutter in the operational program semantics, we introduce a notion of context. A statement context $S$ is a term derived from the grammar $S::=\diamond \mid S$; $s$, where $s \in$ Stmts. We write $S[s]$ for the statement obtained by substituting a statement $s$ for the unique occurrence of $\diamond$ in $S$. Intuitively, a context filled with $s$, e.g., $S[s]$, indicates that $s$ is the next statement to execute in the statement sequence $S[s]$. Similarly, a configuration context $C=\langle g,\langle\ell, S\rangle w, m\rangle$ is a configuration whose top-most frame's statement is replaced with a statement context, and we write $C[s]$ to denote the configuration $\langle g,\langle\ell, S[s]\rangle w, m\rangle$. When $e$ is an expression, we abbreviate $e(C[$ skip $])$ by $e(C)$.

Figure 10 defines the transition relation $\rightarrow^{\mathrm{S}}$ for the standard sequential program statements. The SKIP rule simply steps past the skip statement. The Assume rule proceeds only when the given expression $e$ evaluates to true. The Assign statement stores the value of a given expression in either the local variable 1 or the global variable $\mathrm{g}$. The IF-ThEN and IF-ELSE rules proceeds to either the then or else branch, depending on the current valuation of the given expression $e$. Similarly, the Loop-Do and Loop-End rules proceed to (re-)enter the loop when the given expression $e$ evaluates to true, and step past the loop when $e$ evaluates to false. More interestingly, the CALL rule creates a new procedure frame $f$ by evaluating the given argument $e$, and places $f$ at the top of the 


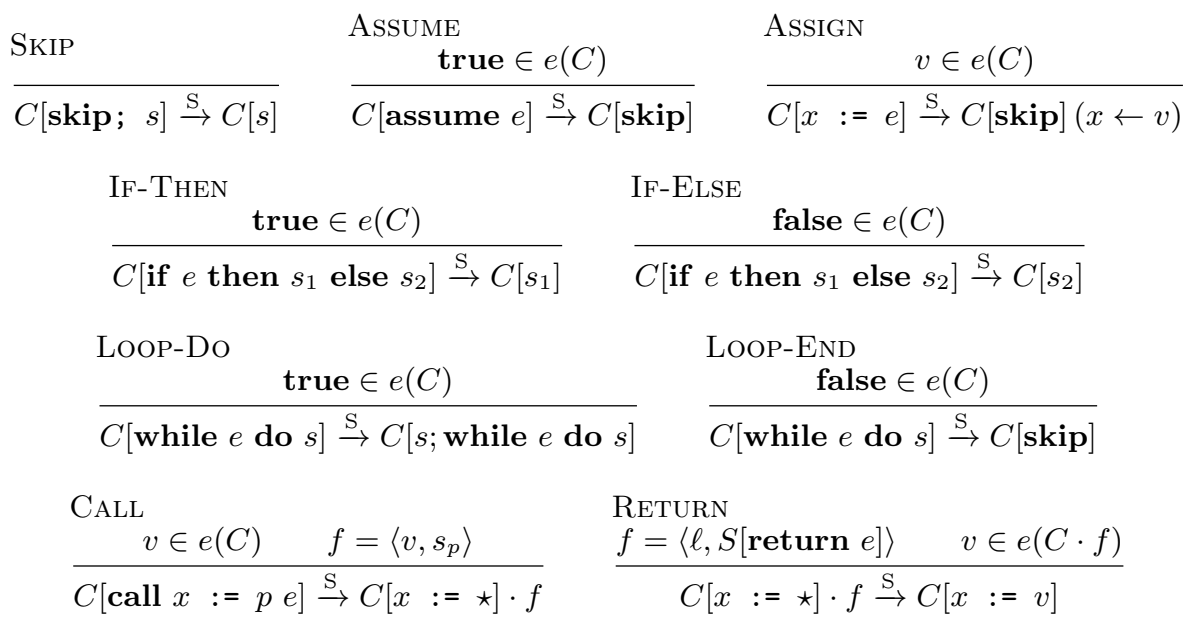

Fig. 10. The transition relation $\rightarrow$ for the standard sequential program statements.

procedure-frame stack. The RETURN rule removes the top-most procedure frame from the stack, and substitutes the valuation of the return expression $e$ into the assignment $x:=\star$ left below by the matching call statement. Note that the transition relation $\rightarrow{ }^{\mathrm{S}}$ is non-deterministic, since the evaluation of an expression $e$ can result in an arbitrary set of possible values. 